\title{
Optimization of Classified Satellite Images using DWT and Fuzzy Logic
}

\author{
Mr. Kaustubh Patil \\ Research Scholar, Vishwakarma Institute of Information Technology Pune India \\ Kauspatil2012@gmail.com
}

\begin{tabular}{|l|l|}
\hline Article History & \multicolumn{1}{c|}{ Abstract } \\
$\begin{array}{l}\text { Article Submission } \\
2 \text { January } 2013 \\
\text { Revised Submission } \\
17 \text { March } 2013 \\
\text { Article Accepted } \\
15 \text { May } 2013 \\
\text { Article Published } \\
30 \text { June 2013 }\end{array}$ & $\begin{array}{l}\text { The image taken by a satellite can be enhanced in terms of its resolution based on } \\
\text { the interpolation can be obtained by DWT. Using DWT, the image at the input is } \\
\text { divided into several sub bands and the speckle noise is also removed. Thereafter, the } \\
\text { high-level images and low-level image at the input can be combined, to produce a } \\
\text { better image applying IDWT. An intermediate stage for approximating high level is } \\
\text { proposed here. The variation in detection approaches for SAR images are done by } \\
\text { using image fusion strategy and novel fuzzy clustering algorithm. To retrieve an } \\
\text { enhanced image, wavelet fusion directives are considered to combine the wavelet } \\
\text { coefficients. A fuzzy C-means algorithm is proposed for identifying the altered and } \\
\text { unaltered regions in the combined difference image. } \\
\text { Keywords - DWT, interpolation, satellite, resolution, enhancement, fuzzy C-means } \\
\text { algorithm, SAR }\end{array}$ \\
\hline
\end{tabular}

\section{Introduction}

Image resolution is always a major concern in several applications, specifically in satellite image resolution enhancement. Hence, a new image enhancement technique which is highly dependent on the combination of the high-level images acquired by DWT and an image at the input [1][2]. The proposed satellite image enhancement method employs DWT to deteriorate the image at the input into several small images. Subsequently the low-level image at the input and high - level images are combined to develop a better image employing IDWT. A midterm stage for approximating high frequency sub images is proposed here resulting in a sharper image [3].

This method effects in four sub images named as low-low (LL), low-high (LH), high-low (HL), and high-high $(\mathrm{HH})$. The whole spectrum of the original image comprises of the above frequency parts.

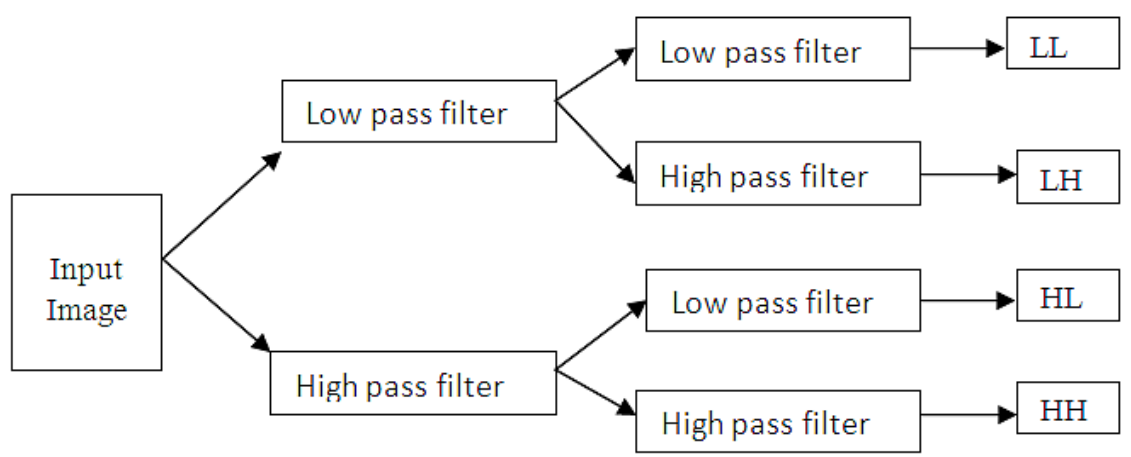

Fig. 1 Schematic representation of DWT filter

Resolution of an image can be magnified through wavelets which is a new technique and proposed several new algorithms for the last decade. Here, we propose a satellite image resolution enhancement technique using combined DWT and the low-resolution information at the input [6]. IDWT helps to combine all the above images to develop the resultant image. Finally, to develop a resolution-enhanced image, an arbitrary approach is used for SAR images which are highly dependent on clustering algorithms [4][5]. 
The image interpolation method is employed to develop a sharper image by using reciprocal data via log-ratio image and mean-ratio image [7]. In order to retrieve the background information and enhanced image, Wavelet combination directives are widely used [8]. For identifying altered and unaltered regions in the combined image, a reconstructed fuzzy $\mathrm{C}$ - means clustering is proposed. The standard of the enhanced image gives the full performance of SAR-image that identifies the change in the detection [9]. In order, we here proposes two major issues to address an arbitrary distribution-free SAR-image that identifies the change in the detection approach in this paper.

\section{Related Work}

Satellite image resolution enhancement has been using several methods until now. Here, we have used two techniques in this paper. They are as follows:

\section{a) CS Based Image Resolution Enhancement}

CS based enhancement technique comprises of two major steps which are as discussed below:

1. Applying WZP, an early estimation to the arbitrary resolution enhanced image is generated.

2. The following tasks are operated by adopting cycle-spinning methodology

- An estimated enhanced image decomposes into many of low level images by removing the high level images.

- Applying WZP to low level images effects in $\mathrm{N}$ high level images.

- The above midway high level images are reconstructed to effect the end image.

\section{b) CWT-Based Image Resolution Enhancement}

The main purpose of DTCWT is to divide into several small images from an image taken at the input. Also, a low-resolution image at the input can decompose into several sub images by using this method. Moreover, all the images are united employing inverse DT-CWT to produce a new enhanced image. Edges in six different directions of high-frequency sub images, is realized by using directional selectivity [10]. For change detection some of the existing methods were used such as K-means, Otsu and FCM. The PCC analysis says that the variation in the detection leads to disastrous by Otsu and K-means. In FCM maps, which is sensitive to noise, decides the need of integrating the information about geographical context. The yielded PCC value is less when compared with proposed method. The computational time required to complete the process is more when compared with our proposed method.

\section{Proposed System and Analysis}

Here, we propose an image resolution-enhancement method which combines DWT images and low-level images. Then, IDWT is applied to unite all the images for a better enhanced image at the end. The standard of the difference image and the exact classification technique gives the full performance of SAR-image which identifies the variation. So an arbitrary SAR-image which identifies the variation approach to mark the following two things in this paper. The block of the proposed system is shown in figure 3 .

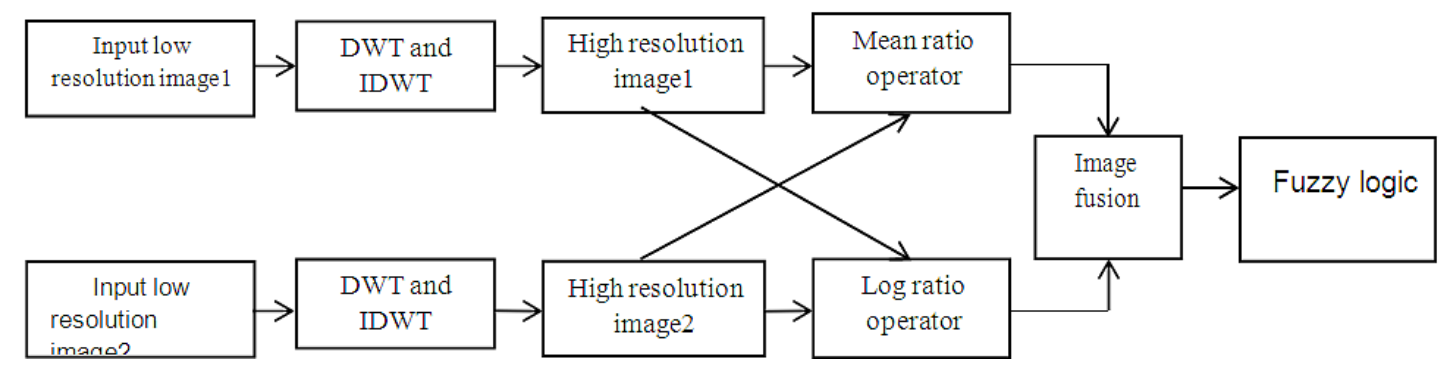

Fig. 3 Schematic representation of the WZP-and CS-based Image super resolution 
The two issues are distinctive which are given below:

- Developing subtracted images by combining a mean-ratio image and a log-ratio image.

- Improving the fuzzy c-means clustering algorithm which is varied in the subtracted image.

The Nasa's Terra first image taken at the Sendai region of Japan is chosen as input image. In this paper, two images taken at same scene at different times is considered. Hence a photo on February 26, 2011(before Tsunami) and March 12, 2011, at 10:30 a.m. (After Tsunami) were taken. The later image is slightly dark and differentiating the changes in coastal area and take the calibration. However, the measuring instrument for flood shows how far flood has extended inland.

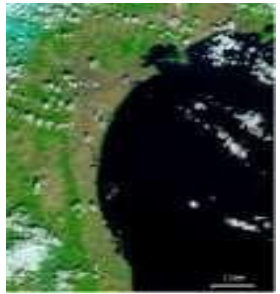

Fig. 4: Input of low resolution image taken at sendai region of japan after Tsunami

If we take care of the edges, the standard of enhanced image will be quite clearer. Hence, DWT algorithm is preferred to protect high-level pixels of the image. All the four images are passed through the combination technique. The LL sub band, with no division is used for the proposed enhancement process as an input. Particularly, low level images are the low frequency parts of the initial image and high level images contain high frequency parts.
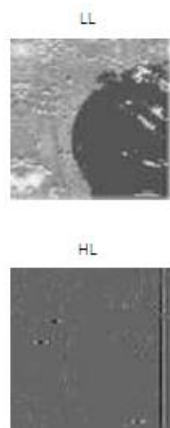
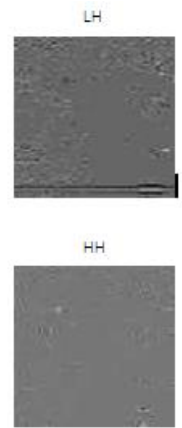

Fig .5 LL, LH, HL, and HH sub bands of a satellite image acquired by using DWT

Hence, apart from operating with low-level images, we are employing the image at the input through the wavelet combining process, which holds low-level information. Therefore, LL sub-band is combined with factor, $\alpha / 2$ to obtain a clearer image. This is shown in fig.6.
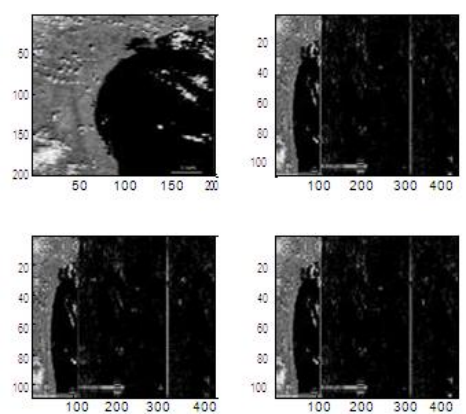

Fig.6 DWT transformed image from MATLAB 
The Speckle noise has a multiplicative tendency which transforms to a noise component in log-ratio component. Moreover, the corresponding ratio image results in enhancing low-level pixels which was proposed in RMD. RMD acts as a tough barrier for speckle noise. It approximates the variations of the image through a mean value. The above two techniques instead of resulting in variation of the detected image in SAR also have many disadvantages like increasing the intensity of low-level pixels and decreasing the intensity of high-level pixels. Hence, the altered and unaltered images from the two techniques should have been more effective. Therefore, approximated high intensity parts of the image are retrieved from subtracted components by intermediate process. This approximation is employed by combining the high-level components and estimating high-level images, using interpolation factor $\alpha / 2$. The midterm process of combining the subtracted image, comprising high-level parts, produces clearer and uncomplicated end image. The final image fusion process is shown in figure 7 .

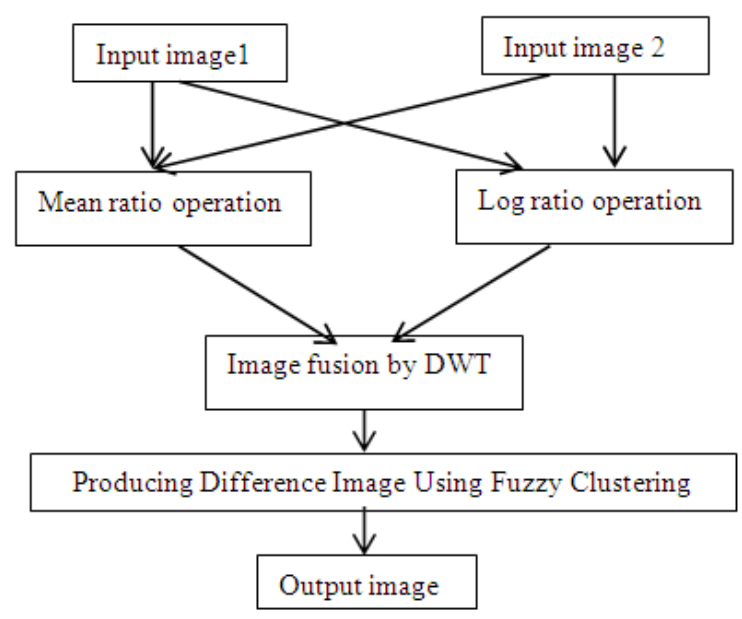

Fig.7 Image fusion process

Fuzzy logic is a probabilistic logic that deals with reasoning that is estimated. on the other side, in a traditional way, binary set will have either 0 or 1 whereas fuzzy logic varies in between 0 and 1 .

Fuzzy c-means clustering algorithm:

1. Allocate values to all $\mathrm{Wi}, \mathrm{j}$.

2. Redo the same.

3. Calculate the midterm of each cluster.

4. Update the fuzzy partition.

5. Until the midterms don't change $\mathrm{Wi}, \mathrm{j}$

Therefore, the varied regions produced by the log-ratio image will not reciprocate the original changed regions to the extreme trends since brighter pixels are in the decrement mode. Concern to RMD, it will deal with low level pixels of temporal images. But the fundamental objective says that, changed areas have high intensity values whereas unchanged regions have low intensity values.

\section{Conclusion}

The quantitative result of PSNR and RMSE shows that our proposed method is robust. The quality of image produced by other interpolation technique is not well suits for satellite images. It produces higher PSNR value. The computational time required to complete the process is less when comparing with other existing methods. The variation in the detection map generated by this technique is robust to noise. The PCC value produced by this method is high when comparing with existing methods. It yields to identify a deal between pixel protection and reducing the noise level. This method takes part in calibrating the local information compared to FLICM. 


\section{References}

[1] M. Ahmed, S. Yamany, N. Mohamed, A. Farag, and T.Moriarty,(Mar. 2002) "A modified fuzzy Cmeans algorithm for bias field estimation and segmentation of MRI data," IEEE Trans. Med. Imag., vol. 21.

[2] W. Cai, S. Chen, and D. Zhang, (Mar. 2007) "Fast and robust fuzzy C-means clustering algorithms incorporating local information for image segmentation," Pattern Recognit., vol. 40.

[3] Atkins, C. A. Bouman, and J. P. Allebach,( Oct. 7- 10, 2001) "Optimal image scaling using pixel classification," in Proc. Int. Conf. Image Process., vol. 3.

[4] Carey, D. B. Chuang, and S. S. Hemami, (Sep. 1999) "Regularity-preserving image interpolation," IEEE Trans. Image Process.,vol. 8.

[5] Demirel, G. Anbarjafari, and S. Izadpanahi, (Aug. 2009), "Improved motionbased localized super resolution technique using discrete wavelet transform for low resolution video enhancement," in Proc. 17th Eur. Signal Process. Conf., Glasgow, Scotland.

[6] J. Inglada and G. Mercier, (May 2007) "A new statistical similarity measure for change detection in multitemporal SAR images and its extension to multiscale change analysis," IEEE Trans. Geosci. Remote Sens., vol. 45.

[7] Fowler, (Mar. 2004 )"The redundant discrete wavelet transform and additive noise,"Mississippi State ERC, Mississippi State University, Tech. Rep. MSSU- COE-ERC-04-04.

[8] J. Inglada and G. Mercier, (May 2007) "A new statistical similarity measure for change detection in multitemporal SAR images and its extension to multiscale change analysis," IEEE Trans. Geosci. Remote Sens., vol. 45.

[9] S. A. Stankevich, S. V. Shklyar and V. M. Tyagur, "Subpixel resolution satellite imaging technique," The International Conference on Digital Technologies 2013, Zilina, 2013, pp. 55-58.

[10] R. Thriveni and Ramashri, "Satellite image enhancement using discrete wavelet transform and threshold decomposition driven morphological filter," 2013 International Conference on Computer Communication and Informatics, Coimbatore, 2013, pp. 1-4 